\title{
Theoretical study of sodium and potassium resonance lines pressure broadened by helium atoms
}

\section{Citation}

Zhu, Cheng, James F. Babb, and Alex Dalgarno. 2006. "Theoretical Study of Sodium and Potassium Resonance Lines Pressure Broadened by Helium Atoms." Physical Review A 73 (1). https://doi.org/10.1103/physreva.73.012506.

\section{Permanent link}

http://nrs.harvard.edu/urn-3:HUL.InstRepos:41417416

\section{Terms of Use}

This article was downloaded from Harvard University's DASH repository, and is made available under the terms and conditions applicable to Other Posted Material, as set forth at http:// nrs.harvard.edu/urn-3:HUL.InstRepos:dash.current.terms-of-use\#LAA

\section{Share Your Story}

The Harvard community has made this article openly available. Please share how this access benefits you. Submit a story. 


\title{
Theoretical Study of Sodium and Potassium Resonance Lines Pressure Broadened by Helium Atoms
}

\author{
Cheng Zhu, James F. Babb and Alex Dalgarno \\ ITAMP, Harvard-Smithsonian Center for Astrophysics, \\ 60 Garden Street, Cambridge, Massachusetts 02138
}

(Dated: January 19, 2006)

\begin{abstract}
We perform fully quantum mechanical calculations in the binary approximation of the emission and absorption profiles of the sodium $3 s-3 p$ and potassium $4 s-4 p$ resonance lines under the influence of a helium perturbing gas. We use carefully constructed potential energy surfaces and transition dipole moments to compute the emission and absorption coefficients at temperatures from 158 to $3000 \mathrm{~K}$. Contributions from quasi-bound states are included. The resulting red and blue wing profiles agree well with previous theoretical calculations and with experimental measurements.
\end{abstract}

PACS numbers: $32.70 . \mathrm{Jz}, 33.70 . \mathrm{Ca}$

\section{INTRODUCTION}

Pressure broadened line profiles of alkali-metal atoms are prominent in the spectra of brown dwarfs and may be a source of opacity in extrasolar giant planet atmospheres $[1,2,3,4,5,6]$. Accurate calculations of these profiles are important in developing effective diagnostics of the temperatures, densities, albedos and composition of the atmospheres of these objects $[7,8]$.

In a recent paper[9], we studied the pressure broadening of the lithium $2 s-2 p$ resonance line. Here we consider the broadening of the $3 s-3 p$ line of sodium and the $4 s-4 p$ line of potassium arising from collisions with helium atoms. We carry out full quantitative quantum-mechanical calculations of the emission and absorption coefficients in the red and blue wings, based on calculated potential energy surfaces and transition dipole moments. We include contributions from quasi-bound states and we allow for the variation of the transition dipole moment with internuclear distance. We compare our results with previous theoretical calculations and experimental measurements.

\section{QUANTUM MECHANICAL CALCULATION}

The system we consider here consists of a mixture of alkali-metal atoms in a bath of helium atoms. If the gas densities are low enough that only binary collisions occur, the problem can be reduced to the radiation of temporarily formed $\mathrm{AHe}(\mathrm{A}=\mathrm{Na}, \mathrm{K})$ molecules. The broadened $n s-n p$ atomic line corresponds to transitions between the excited $\mathrm{A}^{2} \Pi$ and $\mathrm{B}^{2} \Sigma$ electronic states of the AHe molecule and the ground $\mathrm{X}^{2} \Sigma$ state. The $\mathrm{X}^{2} \Sigma$ and $\mathrm{B}^{2} \Sigma$ states have no bound states and the $\mathrm{A}^{2} \Pi$ state has a shallow well which supports a number of bound ro-vibrational levels. Thus we consider both bound-free and free-free transitions. The details of the quantum mechanical calculation of pressure broadening are given in our previous paper [9].

\section{POTENTIALS AND DIPOLE MOMENTS}

We adopt potential energy surfaces computed by Theodorakopoulos and Petsalakis [10] for sodium and by Santra and Kirby [11] for potassium for short and intermediate internuclear distances. The $C_{6}$ coefficients for long range interactions are taken from the latest theoretical calculations of Zhu et al. [12]. The potential energy curves of the $X^{2} \Sigma, A^{2} \Pi$ and $B^{2} \Sigma$ states are shown in Fig. 1 for NaHe and Fig. 2 for KHe. The wavelengths $\lambda(R)$ corresponding to the energy differences $V_{A^{2} \Pi}(R)-V_{X^{2} \Sigma}(R)$ and $V_{B^{2} \Sigma}(R)-V_{X^{2} \Sigma}(R)$ are presented in Figs. 3 and 4 for NaHe and KHe respectively. Extremes in the energy differences as a function of $R$ lead to the presence of satellite features in the emission and absorption spectra. The well-depth of the $\mathrm{A}^{2} \Pi$ state of $\mathrm{NaHe}$ is about three times larger than that of $\mathrm{KHe}$, but both $\mathrm{NaHe}$ and $\mathrm{KHe}$ have six bound vibrational levels for zero angular momentum $J=0$. Table I is a listing of the binding energies of the vibrational levels of the two molecules.

The adopted $X^{2} \Sigma-B^{2} \Sigma$ and $X^{2} \Sigma-A^{2} \Pi$ transition moments [11, 13] are shown in Fig. 5 for NaHe and Fig. 6 for KHe. 


\section{RESULTS AND DISCUSSIONS}

The bound-free emission coefficient is a weighted sum of the emission rates $W_{\nu}$ of the individual ro-vibrational levels of the $\mathrm{A}^{2} \Pi$ state [9]. In Figs. 7 and 8, we plot $W_{\nu}$ as a function of wavelength for a number of ro-vibrational levels for sodium and potassium, respectively. The corresponding radiative lifetimes are listed in Tables II and III. They do not differ much from the lifetimes of the excited atoms, $16.3 \mathrm{~ns}$ for $\mathrm{Na}[14,15]$ and $26.5 \mathrm{~ns}$ for $\mathrm{K}$ [16], into which the $\mathrm{A}^{2} \Pi$ states of the molecules separate with the exception of the $v=4$ level of KHe. For the $v=4$ level of KHe there occurs what appears to be a chance cancellation in the integration of the dipole matrix element.

In Figs. 9 and 10, we present the bound-free contributions to the total emission coefficients in units of $\mathrm{cm}^{-3} \mathrm{~s}^{-1} \mathrm{~Hz}^{-1}$ for sodium and potassium, respectively. The spectra for NaHe and KHe are similar in shape to each other and to LiHe [9] but shifted in wavelength. At low temperatures there occur broad maxima in the emission coefficients at around $670 \mathrm{~nm}$ for $\mathrm{NaHe}$ and $830 \mathrm{~nm}$ for KHe, compared to $870 \mathrm{~nm}$ for LiHe. The emissions are superpositions of the individual ro-vibrational contributions of Figs. 7 and 8, arising from the $\mathrm{A}^{2} \Pi$ states. For the same temperature, the intensity of the red satellite is lower for potassium than for sodium. The emissions decrease rapidly at long wavelengths, because there are no corresponding values of the internuclear distances, as shown by Fig. 3 for sodium and Fig. 4 for potassium.

In the high pressure limit the bound and continuum states are thermally populated and the emission coefficient is the sum of the bound-free and free-free coefficients. In Figs. 11 and 12, we plot our calculated emission coefficients in the high pressure limit for temperatures between 158 and $3000 \mathrm{~K}$ for sodium and potassium, respectively. For sodium, the general shape in the red wing is in agreement with the experimental measurements of York et al. [17] and Havey et al. [18] and the theoretical calculations of Pontius and Sando [19] and Theodorakopoulos and Petsalakis [10]. There is no previous calculation or measurement for potassium. The blue wings are attributed to the $\mathrm{B}^{2} \Sigma-\mathrm{X}^{2} \Sigma$ transitions, where only free-free transitions occur and the resulting profiles decrease rapidly with decreasing wavelength. A weak satellite appears near $532 \mathrm{~nm}$ at $T=3000 \mathrm{~K}$ for sodium and near $708 \mathrm{~nm}$ at $T \approx 2000-3000 \mathrm{~K}$ for potassium, as expected from a consideration of the $\lambda-R$ relations in Figs. 3 and 4 . The satellites are shown on a linear scale in Figs. 13 and 14 .

In Figs. 15 and 16, we plot our calculated absorption coefficients for sodium and potassium, respectively. These profiles decrease steadily with separation from the position of the resonance line except for a blue satellite near $532 \mathrm{~nm}$ at $T \approx 2000-3000 \mathrm{~K}$ for sodium and near $708 \mathrm{~nm}$ at $T \approx 1000-3000 \mathrm{~K}$ for potassium. The general shape in the red and blue wings is in agreement with the theoretical predictions of Allard et al. [7] and Burrows and Volobuyev [8]. Using these calculated absorption profiles, we can infer from the Reid et al. (2000) spectrum [20] of the L5 dwarf, 2MASSW J1507, that the temperature of this dwarf is around $1000 \mathrm{~K}$.

\section{CONCLUSIONS}

We have carried out quantum mechanical calculations for the emission and absorption spectra at wavelengths between 500 and $760 \mathrm{~nm}$ for sodium and between 670 and $930 \mathrm{~nm}$ for potassium at temperatures from 158 to $3000 \mathrm{~K}$. For sodium we find a blue satellite near $532 \mathrm{~nm}$ for $T=3000 \mathrm{~K}$ and a red satellite near $670 \mathrm{~nm}$ for $T \approx 158-240 \mathrm{~K}$ in the emission spectra, and a blue satellite near $532 \mathrm{~nm}$ for $T \approx 2000-3000 \mathrm{~K}$ in the absorption spectra. For potassium we find a blue satellite near $708 \mathrm{~nm}$ for $T \approx 2000-3000 \mathrm{~K}$ and a red satellite near $830 \mathrm{~nm}$ for $T=158 \mathrm{~K}$ in the emission spectra, and a blue satellite near $708 \mathrm{~nm}$ for $T \approx 1000-3000 \mathrm{~K}$ in the absorption spectra. Our results are in good agreement with previous calculations and experiment. We infer that the temperature of the brown dwarf, 2MASSW J1507, is around $1000 \mathrm{~K}$.

\section{Acknowledgments}

This work was supported in part by the NSF through a grant for ITAMP to the Smithsonian Astrophysical Observatory and Harvard University and by NASA under award NAG5-12751.

[1] R. Jayawardhana, Science 303, 322 (2004).

[2] K. Lodders, Science 303, 323 (2004).

[3] A. Burrows, Nature 433, 261 (2005).

[4] A. Burrows, W. B. Hubbard, J. I. Lunine, and J. Liebert, Rev. Mod. Phys. 73, 719 (2001). 
[5] S. Seager and D. D. Sasselov, Astrophys. J. 537, 916 (2000).

[6] T. M. Brown, Astrophys. J. 553, 1006 (2001).

[7] N. F. Allard et al., Astron. Astrophys. 411, L473 (2003).

[8] A. Burrows and M. Volobuyev, Astrophys. J. 583, 985 (2003).

[9] C. Zhu, J. F. Babb and A. Dalgarno, Phys. Rev. A 71, 052710 (2005).

[10] G. Theodorakopoulos and I. D. Petsalakis, J. Phys. B 26, 4367 (1993).

[11] R. Santra and K. Kirby, Phys. Rev. A, in press (2005).

[12] C. Zhu, A. Dalgarno, S. G. Porsev and A. Derevianko, Phys. Rev. A 70, 032722 (2004).

[13] P. C. Stancil, private communication.

[14] U. Volz, M. Majerus, H. Liebel, A. Schmitt and H. Schmoranzer, Phys. Rev. Lett. 76, 2862 (1996).

[15] U. Volz and H. Schmoranzer, Phys. Scr. T65, 48 (1996).

[16] H. Wang et al., Phys. Rev. A 55, R1569 (1997).

[17] G. York, R. Scheps and A. Gallagher, J. Chem. Phys. 63, 1052 (1975).

[18] M. D. Havey, S. E. Frolking and J. J. Wright, Phys. Rev. Lett. 45, 1783 (1980).

[19] E. M. Pontius and K. M. Sando, Phys. Rev. A 28, 3117 (1983).

[20] I. N. Reid et al., Astron. J. 119, 369 (2000). 
TABLE I: Vibrational energy levels $\left(\mathrm{cm}^{-1}\right)$ of the $\mathrm{A}^{2} \Pi$ state of $\mathrm{NaHe}$ and KHe molecules.

\begin{tabular}{c|cccccc}
\hline \hline$v$ & 0 & 1 & 2 & 3 & 4 & 5 \\
\hline$E^{v, J=0}, \mathrm{NaHe}$ & -512.9 & -317.2 & -169.9 & -83.78 & -35.26 & -2.130 \\
$E^{v, J=0}, \mathrm{KHe}$ & -163.7 & -90.45 & -44.82 & -19.11 & -7.034 & -1.037 \\
\hline \hline
\end{tabular}


TABLE II: Lifetimes (ns) of selected ro-vibrational levels $(v, J)$ of the $\mathrm{NaHe} \mathrm{A}^{2} \Pi$ state.

\begin{tabular}{c|cccccc}
\hline \hline & $v=0$ & $v=1$ & $v=2$ & $v=3$ & $v=4$ & $v=5$ \\
\hline$J=0$ & 22.8 & 21.2 & 19.6 & 17.9 & 16.9 & 16.4 \\
$J=5$ & 22.7 & 21.2 & 19.5 & 17.7 & 16.8 & \\
$J=10$ & 22.5 & 21.0 & 19.1 & 17.3 & & \\
$J=18$ & 22.0 & 20.2 & & & & \\
\hline \hline
\end{tabular}


TABLE III: Lifetimes (ns) of selected ro-vibrational levels $(v, J)$ of the KHe $\mathrm{A}^{2} \Pi$ state.

\begin{tabular}{c|cccccc}
\hline \hline & $v=0$ & $v=1$ & $v=2$ & $v=3$ & $v=4$ & $v=5$ \\
\hline$J=0$ & 28.3 & 26.6 & 25.2 & 26.2 & 79.8 & 30.0 \\
$J=5$ & 28.2 & 26.4 & 25.1 & 27.1 & 106.9 & \\
$J=10$ & 27.9 & 26.1 & 24.6 & & & \\
$J=15$ & 27.4 & & & & & \\
\hline \hline
\end{tabular}



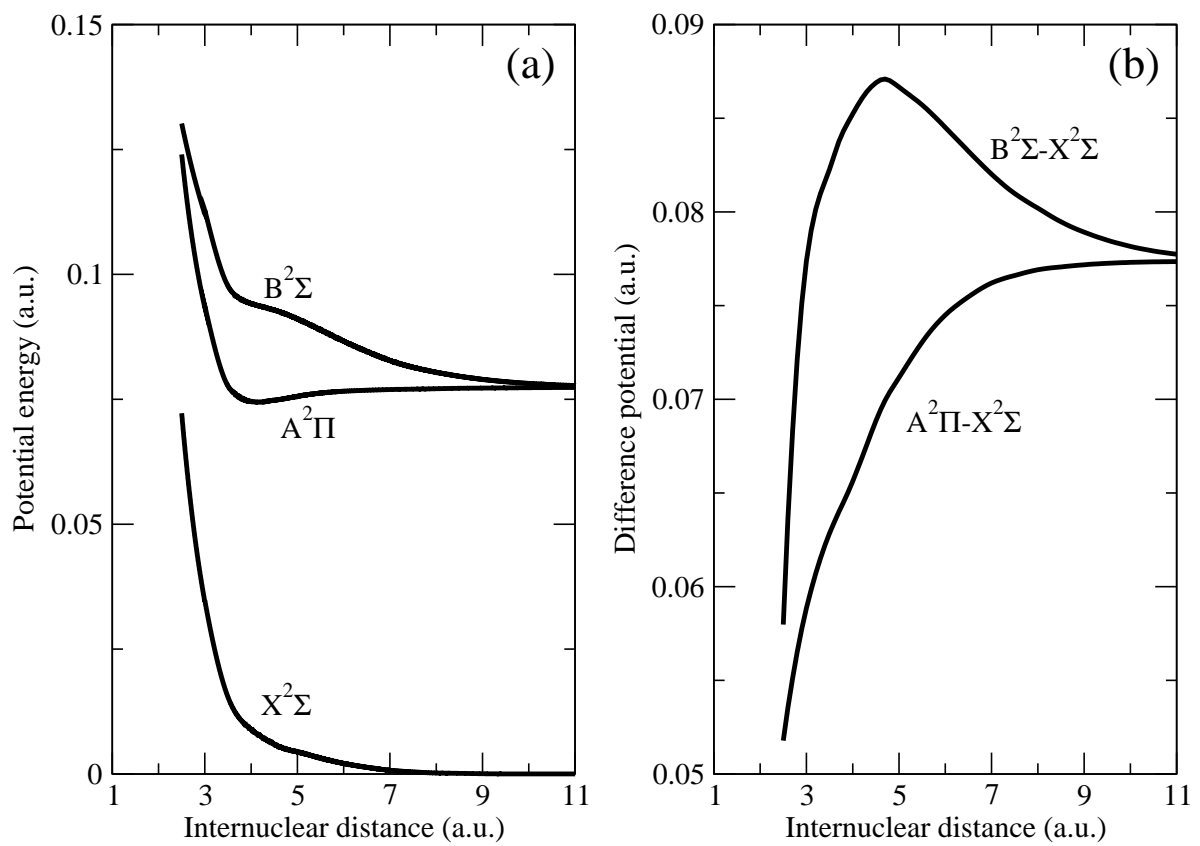

FIG. 1: (a) Adopted potentials $V(R)$ for the NaHe $X^{2} \Sigma, A^{2} \Pi$ and $B^{2} \Sigma$ states. (b) Difference potentials $V_{A^{2} \Pi}(R)-V_{X^{2} \Sigma}(R)$ and $V_{B^{2} \Sigma}(R)-V_{X^{2} \Sigma}(R)$. 

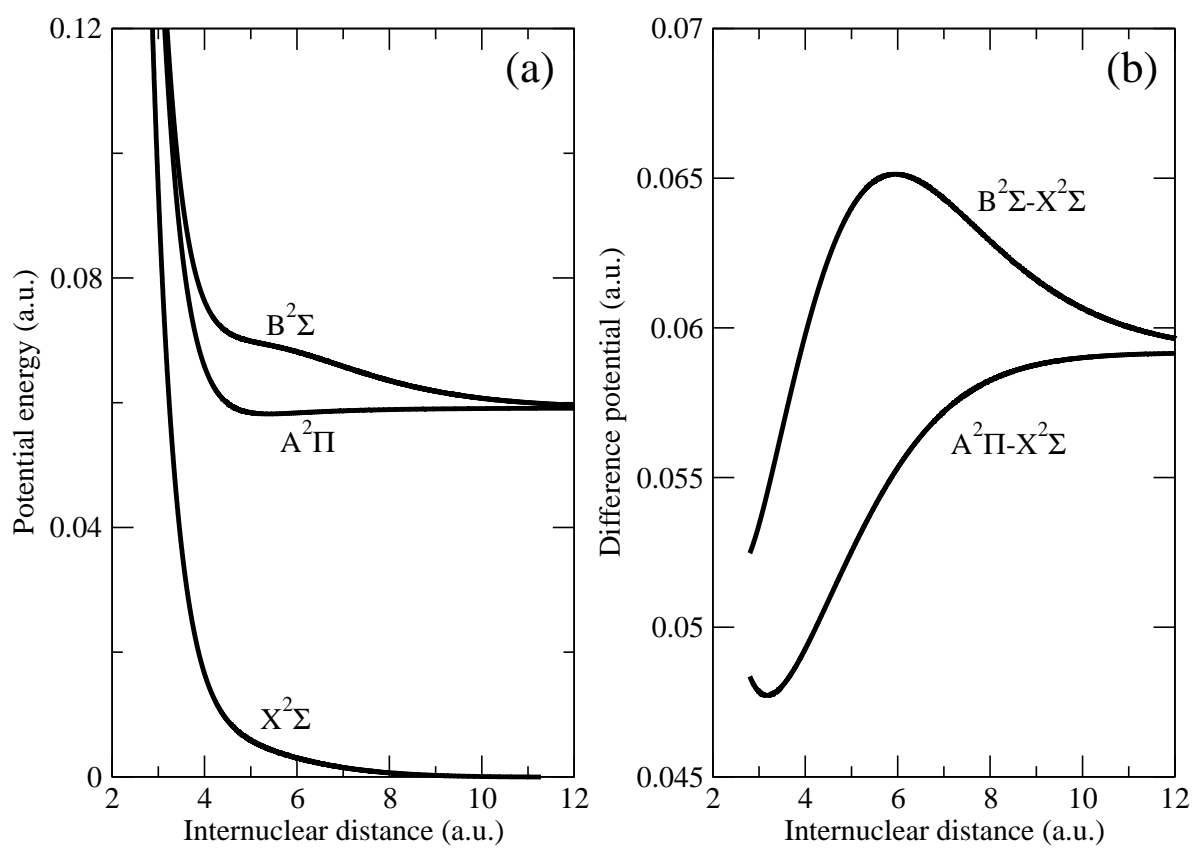

FIG. 2: (a) Adopted potentials $V(R)$ for the KHe $X^{2} \Sigma, A^{2} \Pi$ and $B^{2} \Sigma$ states. (b) Difference potentials $V_{A^{2} \Pi}(R)-V_{X^{2} \Sigma}(R)$ and $V_{B^{2} \Sigma}(R)-V_{X^{2} \Sigma}(R)$. 


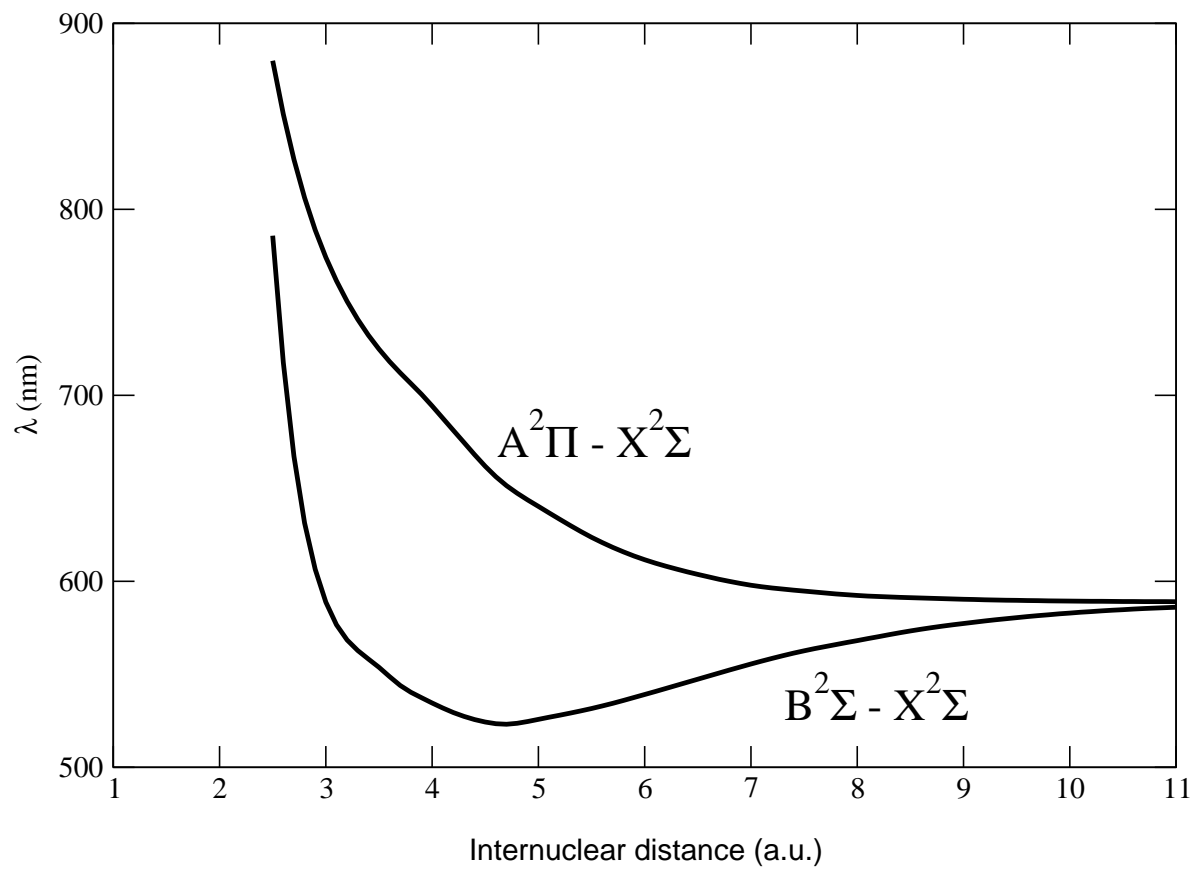

FIG. 3: Wavelengths corresponding to difference potentials for $\mathrm{NaHe} \mathrm{A}^{2} \Pi-\mathrm{X}^{2} \Sigma$ and $\mathrm{B}^{2} \Sigma-\mathrm{X}^{2} \Sigma$ transitions, respectively. 


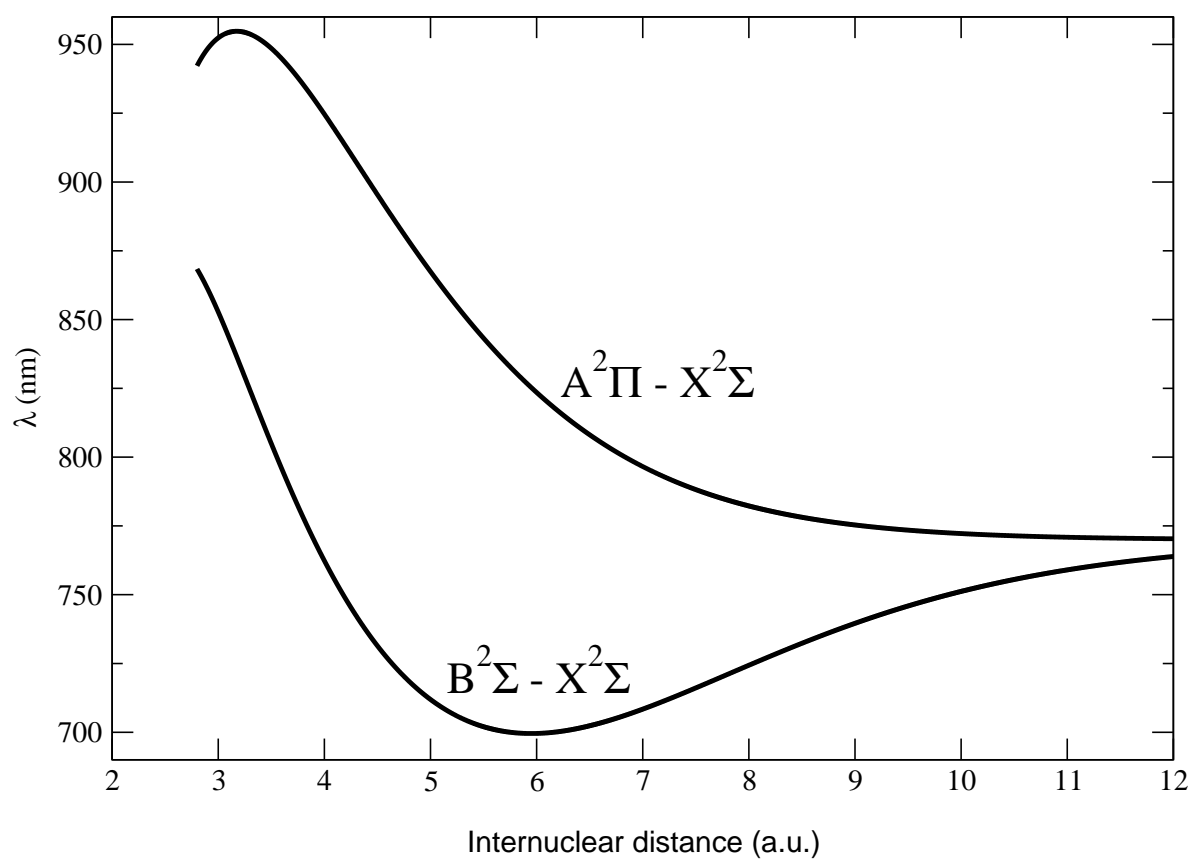

FIG. 4: Wavelengths corresponding to difference potentials for KHe $\mathrm{A}^{2} \Pi-\mathrm{X}^{2} \Sigma$ and $\mathrm{B}^{2} \Sigma-\mathrm{X}^{2} \Sigma$ transitions, respectively. 


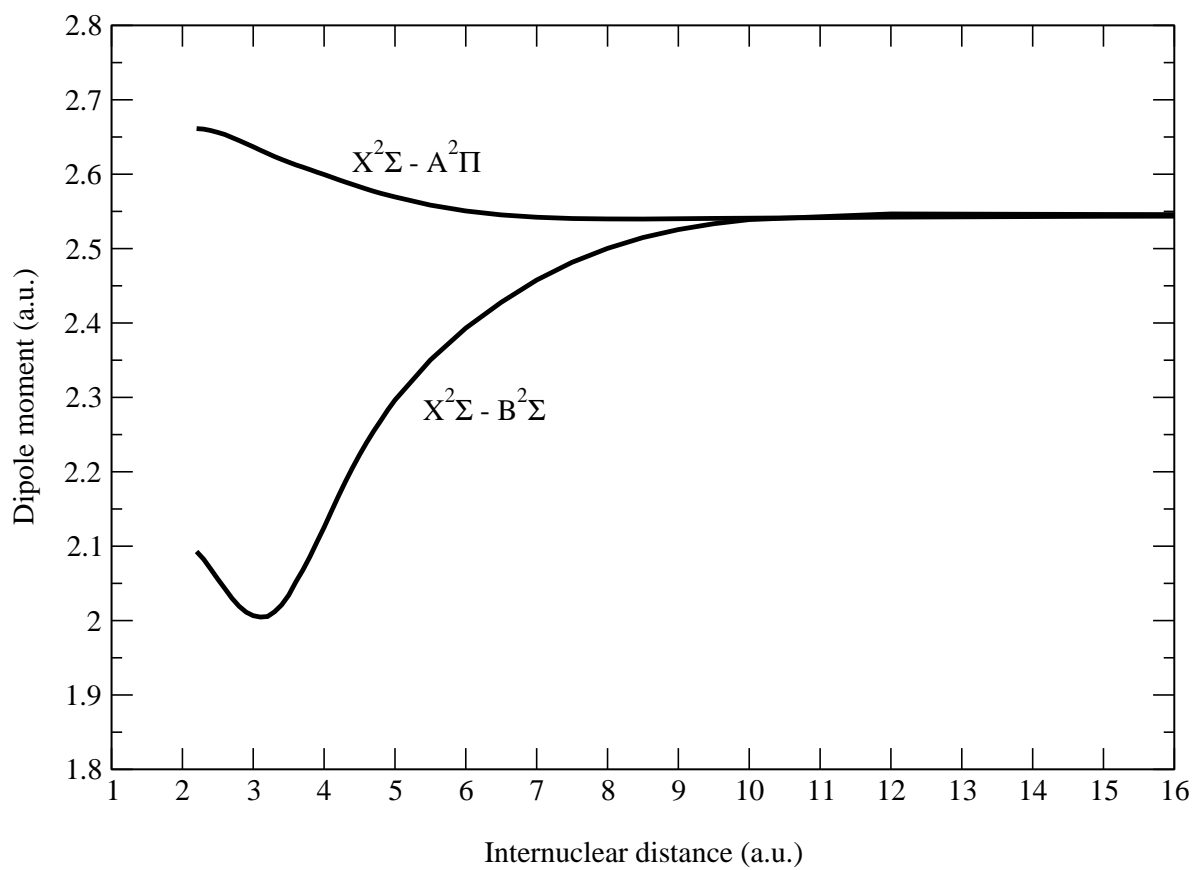

FIG. 5: Adopted dipole moment curves for the NaHe $X^{2} \Sigma-A^{2} \Pi$ and $X^{2} \Sigma-B^{2} \Sigma$ transitions. 


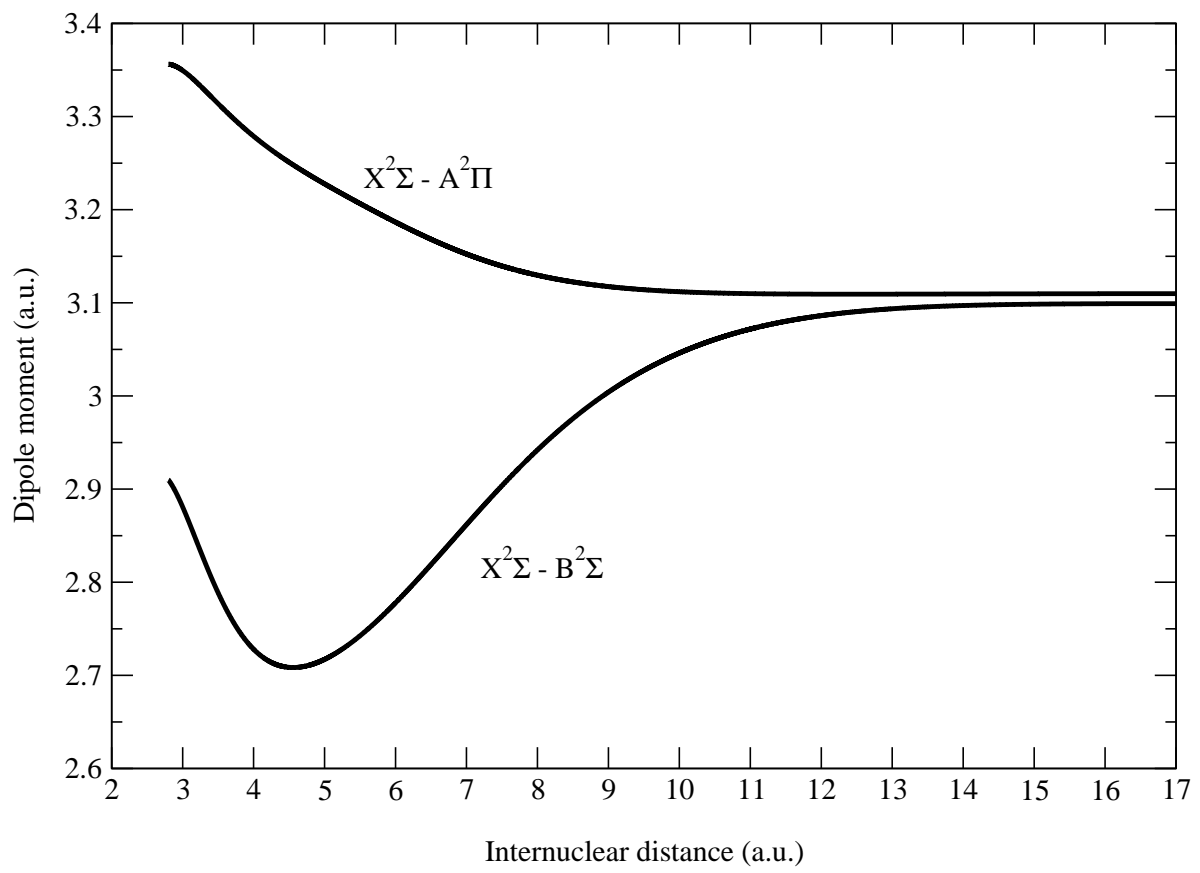

FIG. 6: Adopted dipole moment curves for the KHe $X^{2} \Sigma-A^{2} \Pi$ and $X^{2} \Sigma-B^{2} \Sigma$ transitions. 


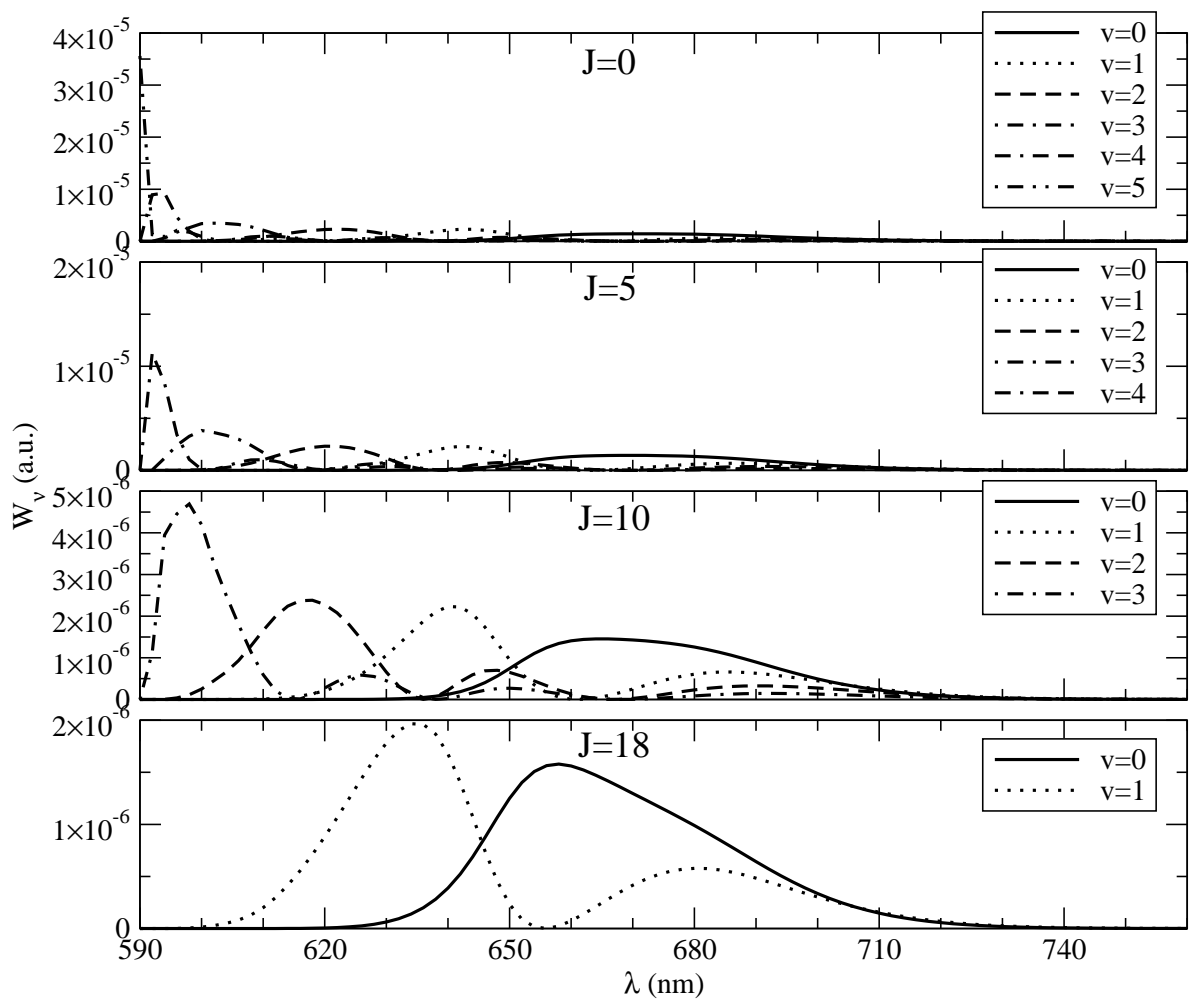

FIG. 7: $W_{\nu}$ for vibrational levels $v=0-5$ for $J=0$ (upper panel), 5 (upper middle panel), 10 (lower middle panel) and 18 (lower panel) for the NaHe system. 


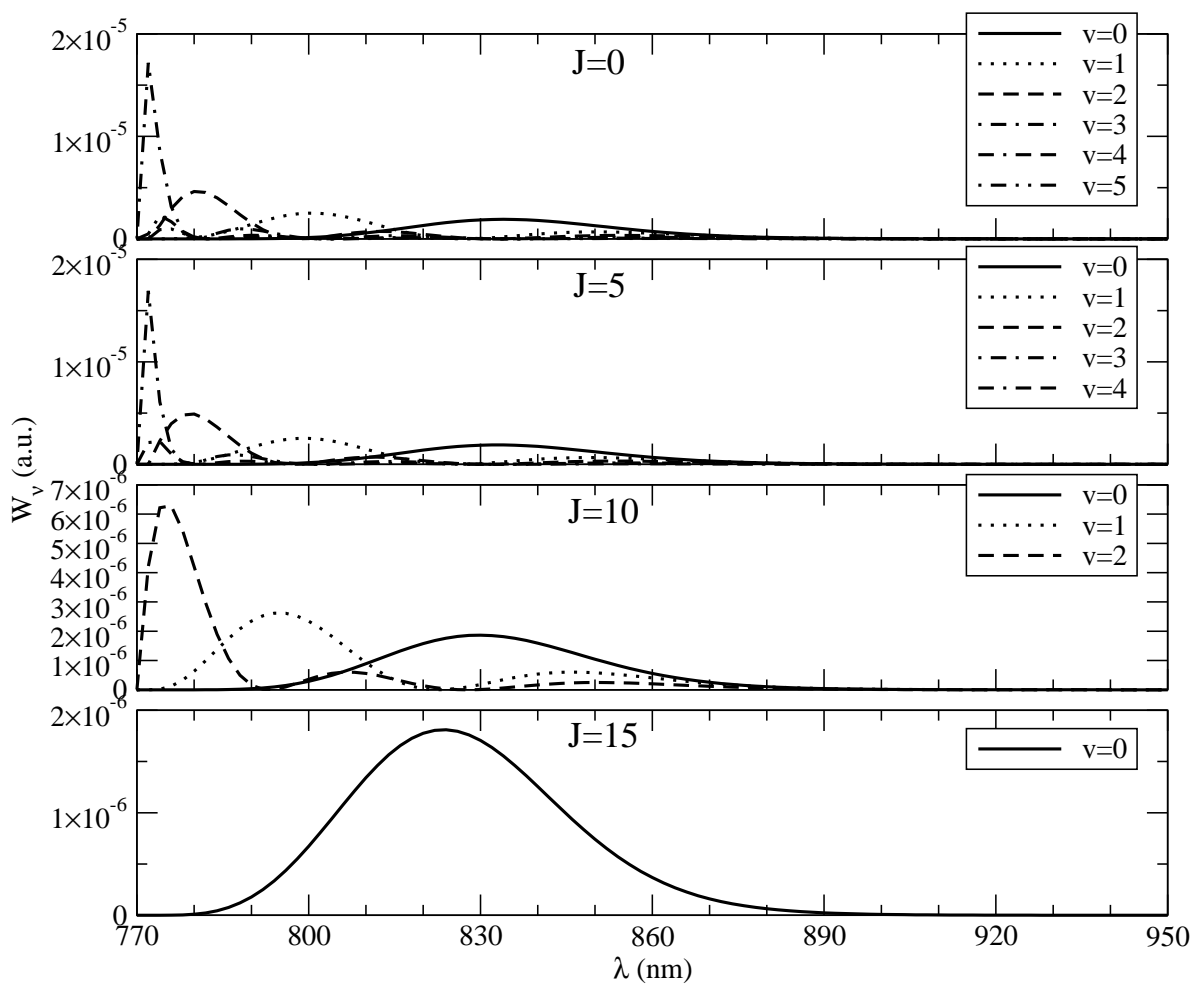

FIG. 8: $W_{\nu}$ for vibrational levels $v=0-5$ for $J=0$ (upper panel), 5 (upper middle panel), 10 (lower middle panel) and 15 (lower panel) for the KHe system. 


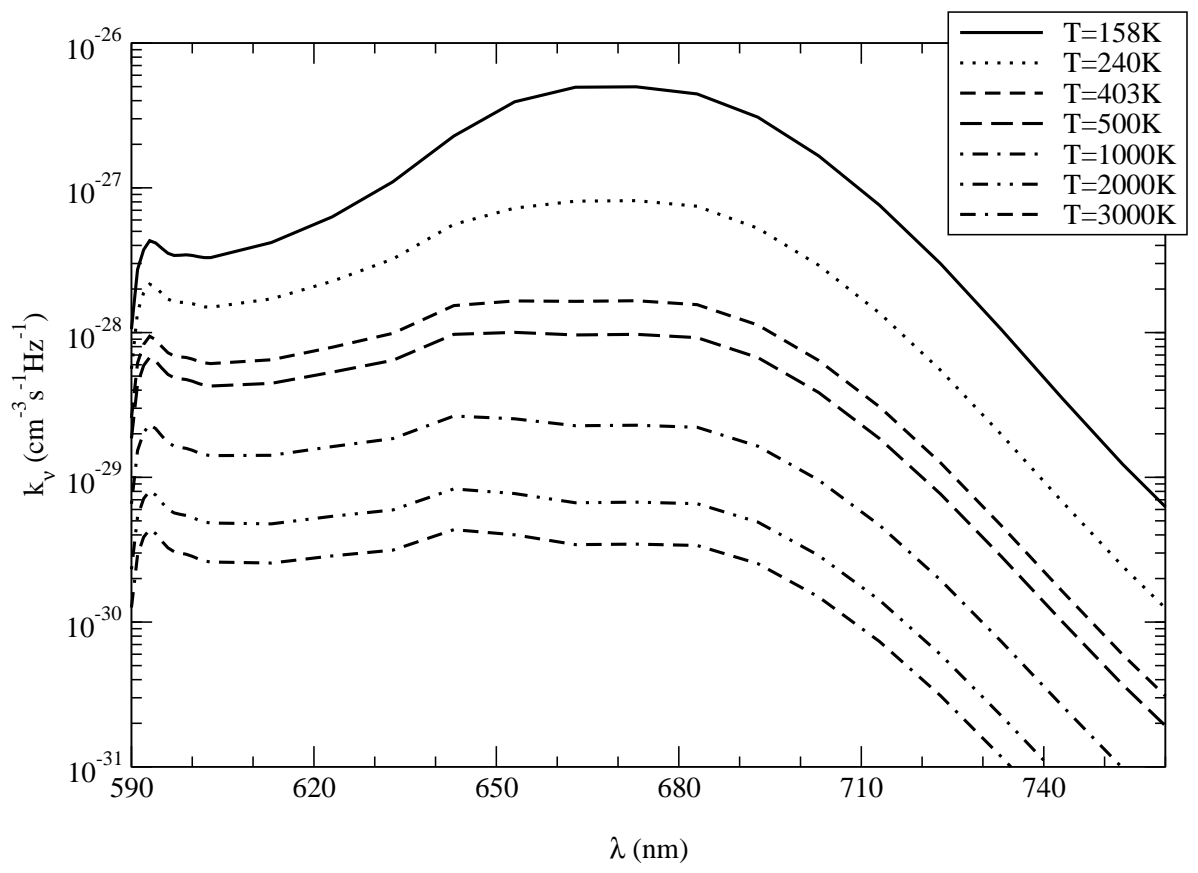

FIG. 9: Contributions of bound-free transitions to the total emission coefficients of NaHe at temperatures $T=158,240,403$, 500, 1000, 2000 and 3000K. Unit gas densities are used, $n_{N a}=n_{H e}=1 \mathrm{~cm}^{-3}$. 


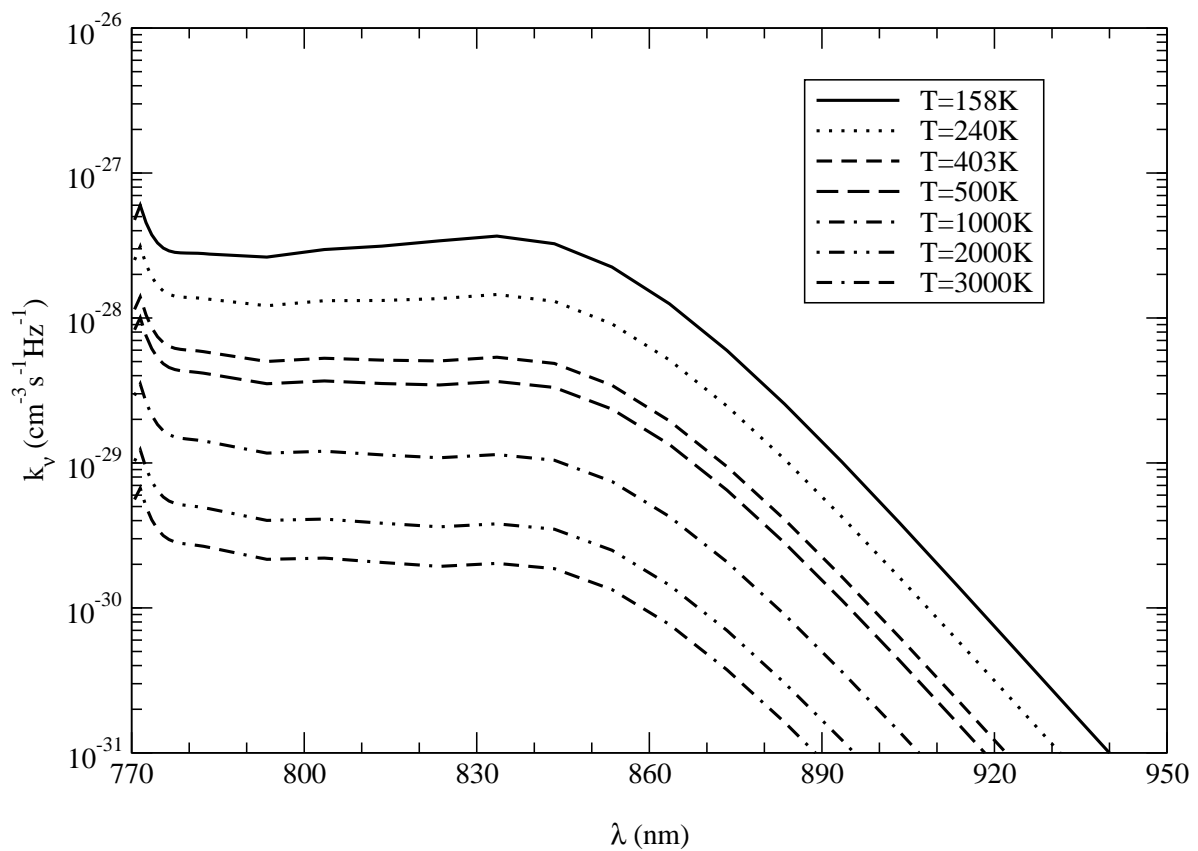

FIG. 10: Contributions of bound-free transitions to the total emission coefficients of KHe at temperatures $T=158,240,403$, 500, 1000, 2000 and 3000K. Unit gas densities are used, $n_{K}=n_{H e}=1 \mathrm{~cm}^{-3}$. 


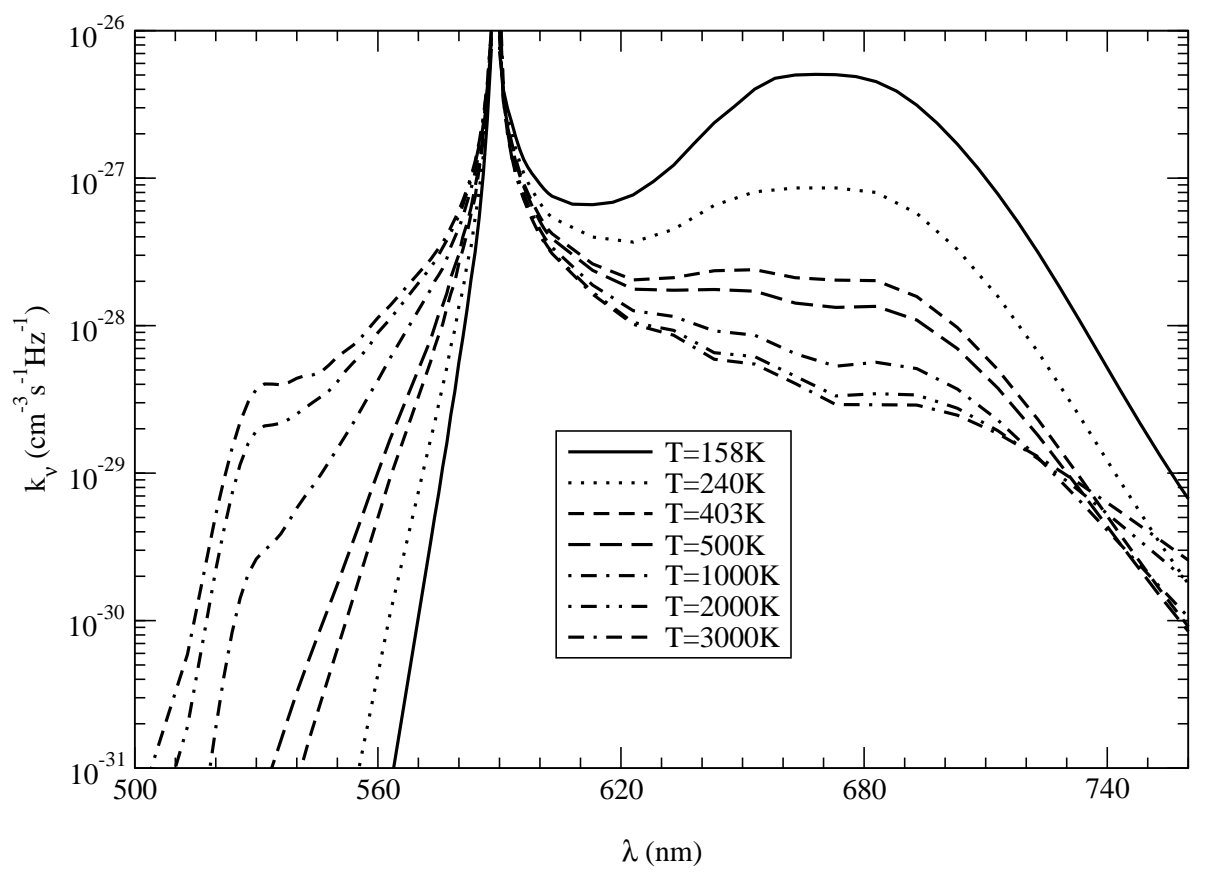

FIG. 11: High pressure emission coefficients of NaHe at temperatures $T=158,240,403,500,1000,2000$ and $3000 \mathrm{~K}$. Unit gas densities are used, $n_{N a}=n_{H e}=1 \mathrm{~cm}^{-3}$. 


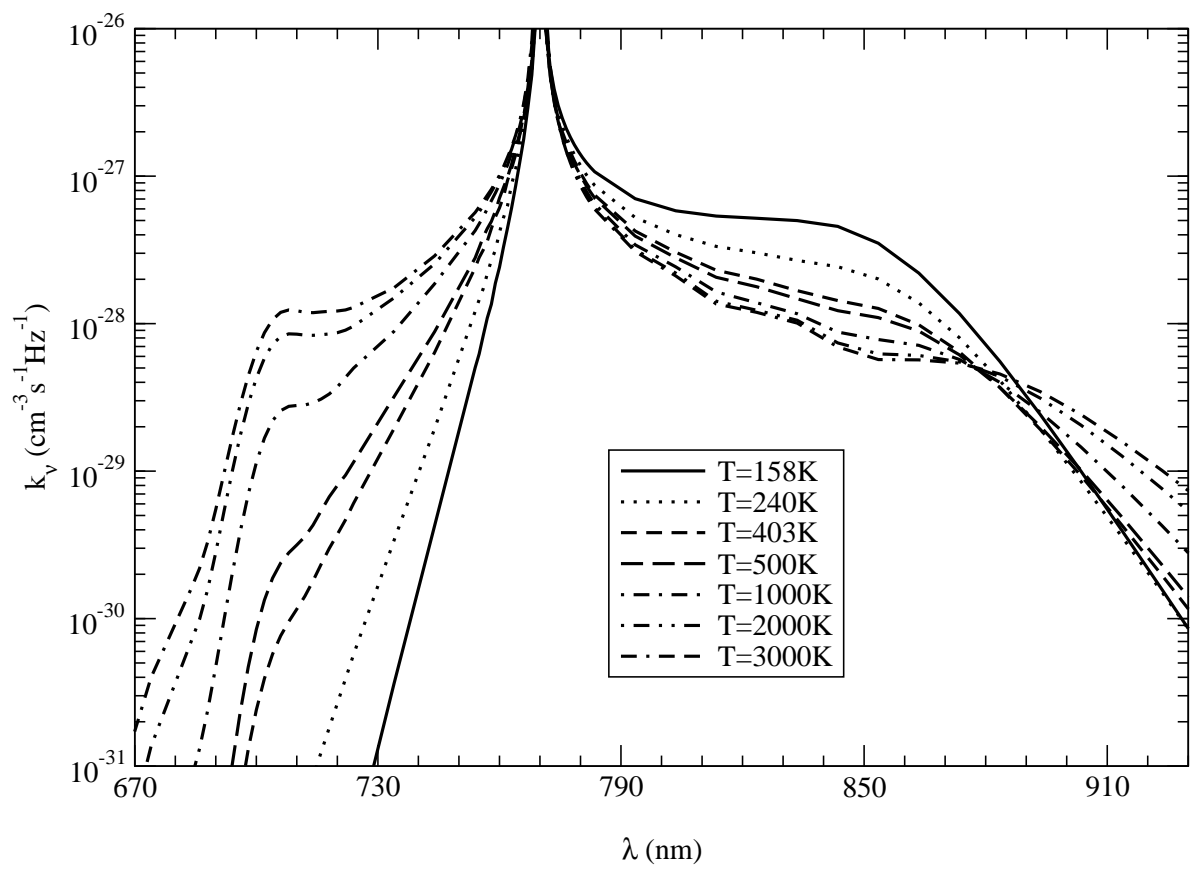

FIG. 12: High pressure emission coefficients of KHe at temperatures $T=158,240,403,500,1000,2000$ and 3000K. Unit gas densities are used, $n_{K}=n_{H e}=1 \mathrm{~cm}^{-3}$. 


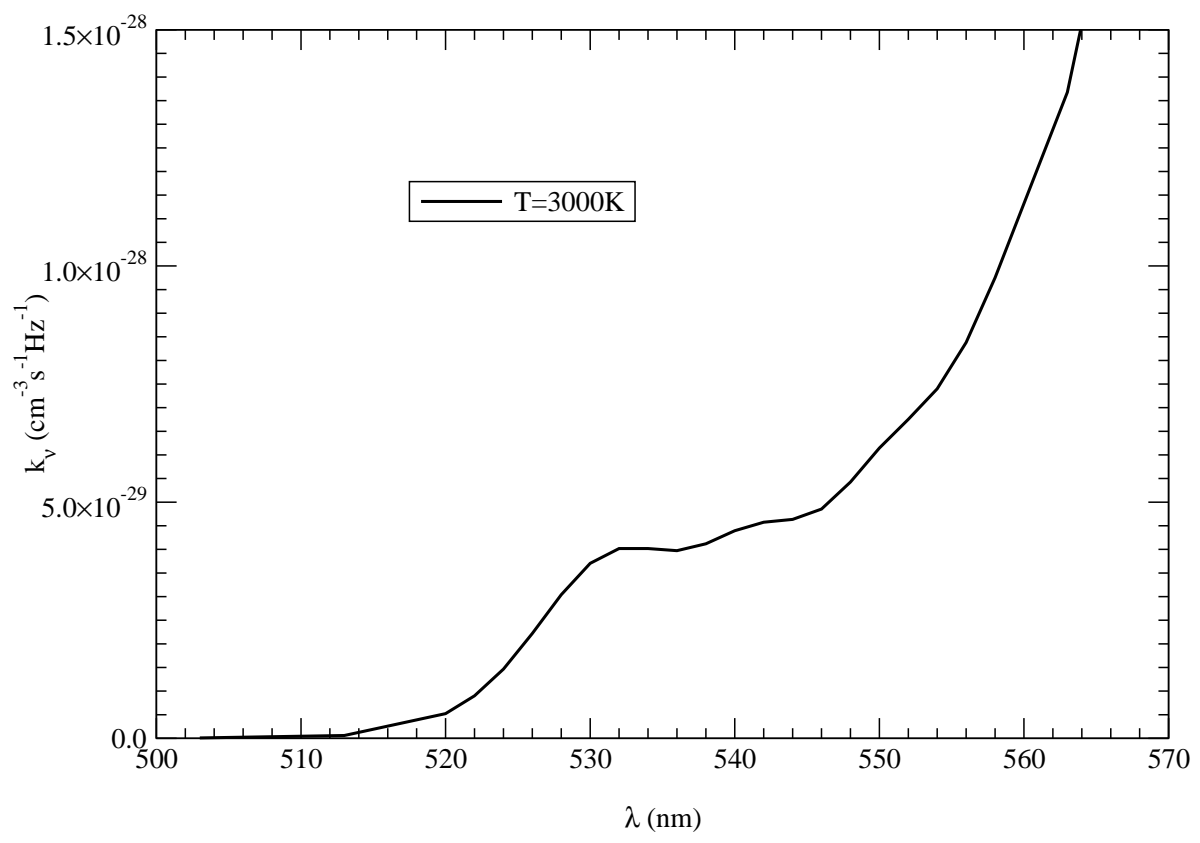

FIG. 13: Emission coefficients of NaHe at temperature $T=3000 \mathrm{~K}$ plotted on a linear scale. Unit gas densities are used, $n_{N a}=n_{H e}=1 \mathrm{~cm}^{-3}$. 


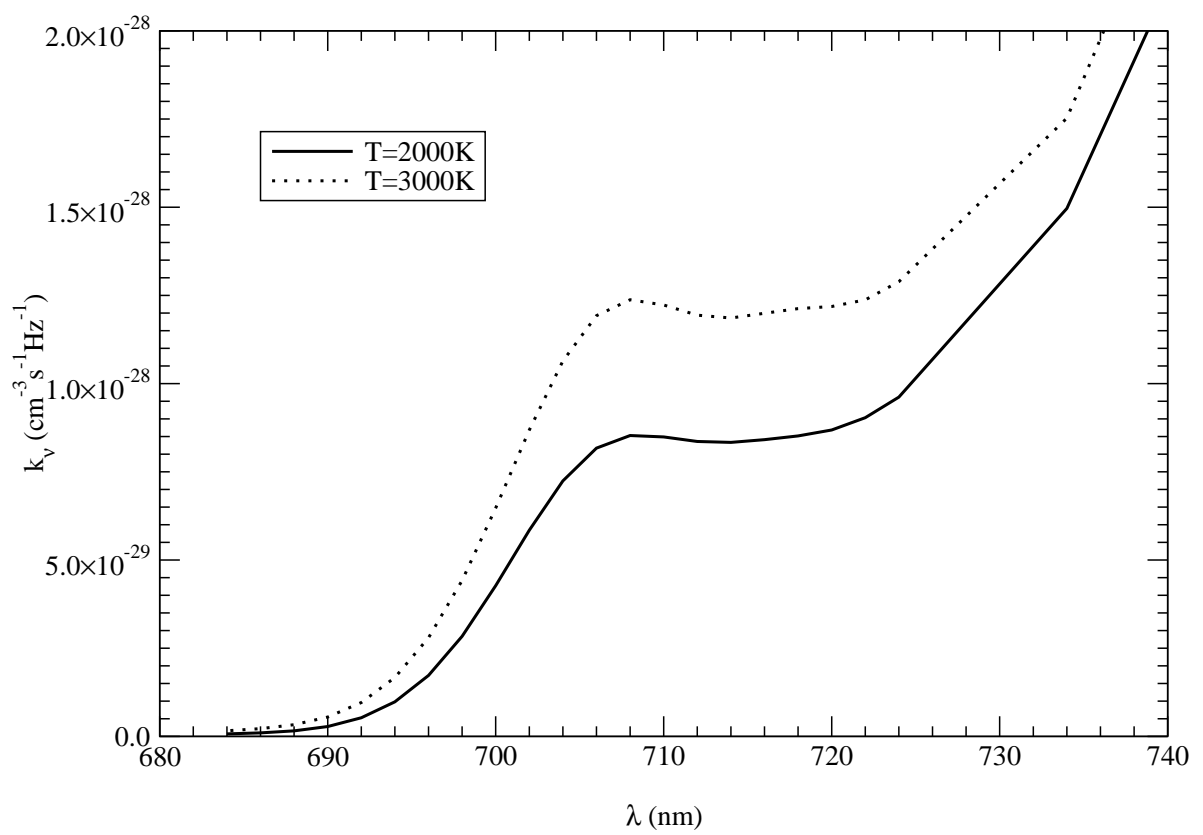

FIG. 14: Emission coefficients of KHe at temperatures $T=2000$ and $3000 \mathrm{~K}$ plotted on a linear scale. Unit gas densities are used, $n_{K}=n_{H e}=1 \mathrm{~cm}^{-3}$. 


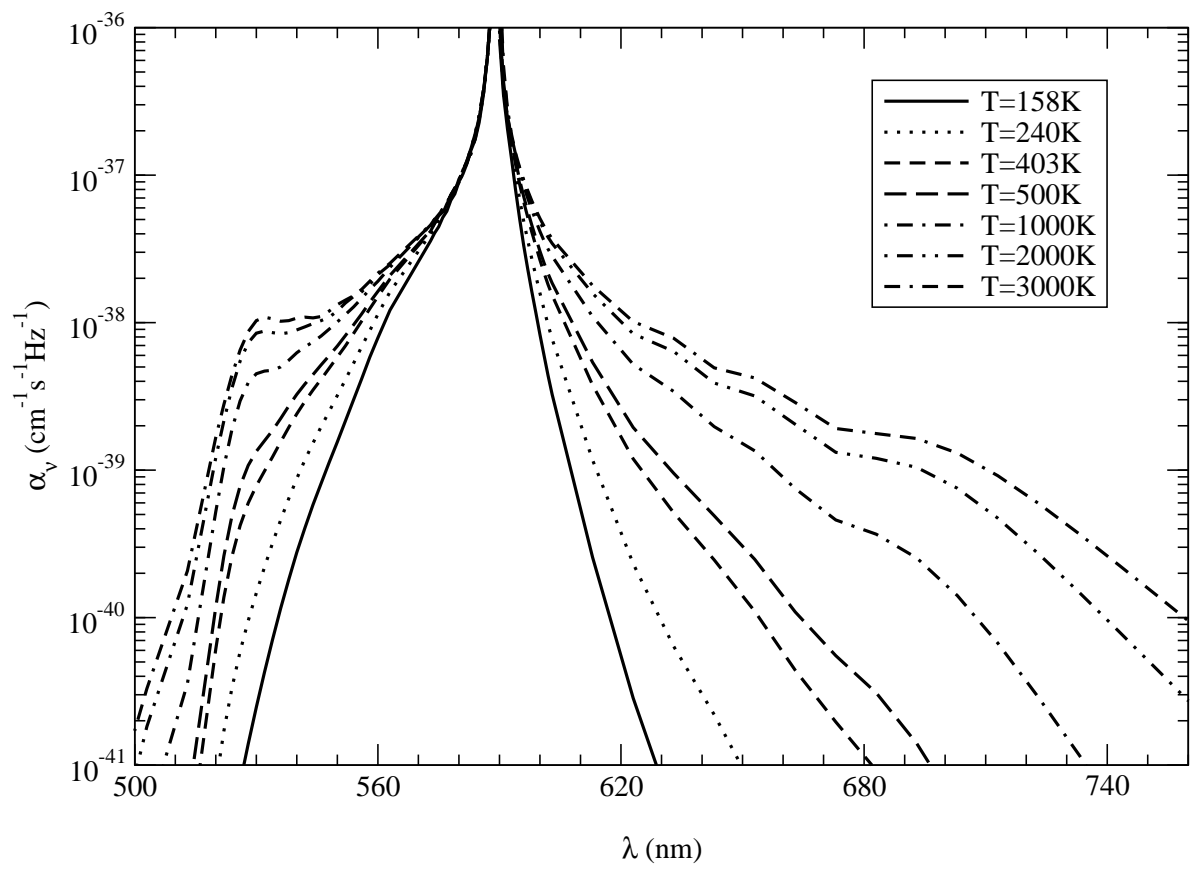

FIG. 15: Absorption coefficients of NaHe at temperatures $T=158,240,403,500,1000,2000$ and $3000 \mathrm{~K}$. Unit gas densities are used, $n_{N a}=n_{H e}=1 \mathrm{~cm}^{-3}$. 


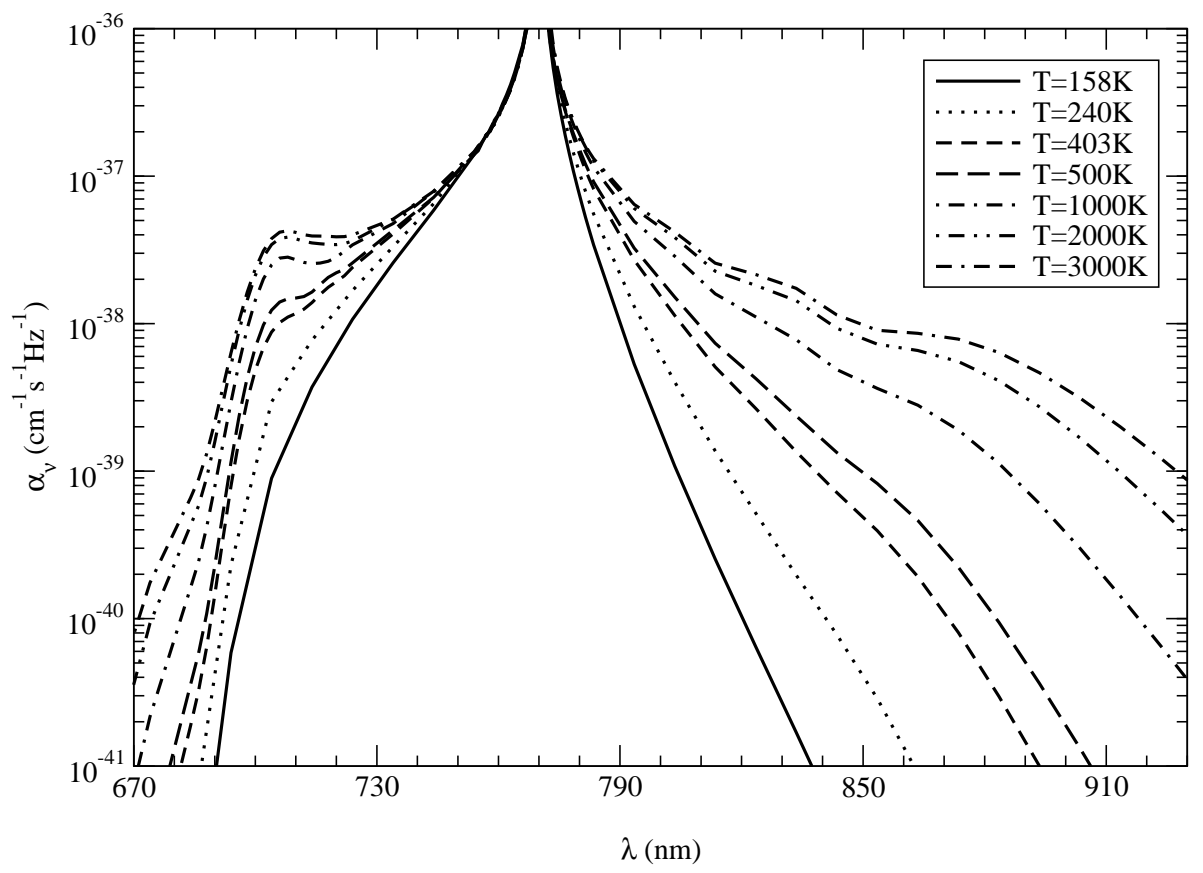

FIG. 16: Absorption coefficients of KHe at temperatures $T=158,240,403,500,1000,2000$ and 3000K. Unit gas densities are used, $n_{K}=n_{H e}=1 \mathrm{~cm}^{-3}$. 